\title{
Oral findings in Rett syndrome: A systematic review of the dental literature
}

\author{
María-Cristina Fuertes-González ${ }^{1}$, Francisco-Javier Silvestre ${ }^{2}$, José-Manuel Almerich-Silla ${ }^{3}$
}

${ }^{1}$ Staff dentist of the Red Cross Dental Clinic for Special Patients, Valencia. Professor of the Master in Hospital Odontology and Special Patients

${ }^{2}$ Assistant Professor of Odontology in Special Patients. Department of Stomatology, University of Valencia. Director of the Master in Hospital Odontology and Special Patients

${ }^{3}$ Assistant Professor of Preventive and Community Care Odontology. Department of Stomatology, University of Valencia (Spain)

Correspondence:

Departament d'Estomatologia

Clinica Odontològica

Universitat de València

Gascó Oliag 1

46010 - Valencia (Spain)

francisco.silvestre@uv.es

Received: $27 / 02 / 2010$

Accepted: $14 / 04 / 2010$

\author{
Fuertes-González MC, Silvestre FJ, Almerich-Silla JM. Oral findings in \\ Rett syndrome: A systematic review of the dental literature. Med Oral \\ Patol Oral Cir Bucal. 2011 Jan 1;16 (1):e37-41. \\ http://www.medicinaoral.com/medoralfree01/v16i1/medoralv16ilp37.pdf

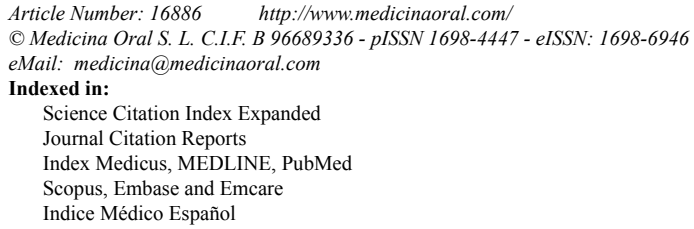

\begin{abstract}
Rett syndrome (RS) is a chromosome X-linked genetic neurological disorder characterized by developmental regression, particularly in relation to expressive language and use of the hands, together with profound mental retardation, that almost exclusively affects females. The present review describes the 35 cases of RS Publisher in the indexed literature (Medline) - the first corresponding to 1985 and the last to the year 2007. Certain oral manifestations of the disease are derived from the drug treatment prescribed to control the disease, while others are common to other clinical conditions characterized by convulsion activity, difficulties for correct oral hygiene, walking problems and/or an excess of oral / digital-manual habits. In any case, bruxism is the oral habit most frequently associated with RS - the treatment of which remains the subject of controversy.
\end{abstract}

Key words: Rett syndrome, oral manifestations, bruxism, review.

\section{Introducción}

Rett syndrome (RS) is a neurological development disorder characterized by the regression of psychomotor development with autistic manifestations, decelerated growth of the head, seizures, and stereotyped repetitive movements of the hands (1). Although initially described by Rett in 1966 (2), the compilation of 35 cases describing the clinical features of the syndrome was published by Hagberg et al. in 1983 (3).

RS almost exclusively affects females, since it involves a dominant X chromosome-linked mutation (MECP2 gene, region Xq28) (4). Although initially regarded as a neurodegenerative disease, it is now known that RS does not involve neuron death (5).

The diagnosis of classical RS is based on a series of clinical criteria (table 1) (6), since MECP2 gene mutations alone are not equivalent to a diagnosis of RS, and can also be seen in other conditions such as autism, for example. A study of RS in the Spanish population published in 1999 (7) documented a total of 207 cases, though the true figure must be greater, since the epidemiological studies made in different countries yield a prevalence of between $1 / 12,000(0.65 / 10,000)$ and $1 / 15,000$ $(0.41 / 10,000)$. 
Table 1. Reviewed diagnostic criteria in Rett syndrome (6).

\begin{tabular}{|c|c|}
\hline \multicolumn{2}{|c|}{ Required criteria } \\
\hline 1. & An apparently normal pre- and perinatal period. \\
\hline 2. & Mostly normal psychomotor development up to 6 months of age. \\
\hline 3. & Normal head circumference at birth. \\
\hline 4. & Deceleration of head growth. \\
\hline 5. & Loss of manual functional skills at age $0.5-2.5$ years. \\
\hline 6. & $\begin{array}{l}\text { Stereotypic movements of the hands, such as clenching, applause, washing movements } \\
\text { or taking the hands to the mouth. }\end{array}$ \\
\hline 7. & $\begin{array}{l}\text { Social retraction, communication dysfunction, forgetting of learned words, and } \\
\text { cognitive disability. }\end{array}$ \\
\hline 8. & Gait apraxia or failure to learn to walk. \\
\hline \multicolumn{2}{|c|}{ Supporting criteria } \\
\hline & $\begin{array}{l}\text { Respiratory dysfunction: apneas, hyperventilation, sharp expulsion of air and saliva, } \\
\text { aerophagia. }\end{array}$ \\
\hline 2. & Bruxism. \\
\hline 3. & Sleep disturbances from infancy. \\
\hline 4. & Abnormal muscle tone associated to dystonia \\
\hline 5 . & Peripheral vasomotor alterations. \\
\hline 6. & Scoliosis / kyphosis. \\
\hline 7. & Retarded growth. \\
\hline 8. & Small, hypotrophic and cold feet; small and thin hands. \\
\hline \multicolumn{2}{|c|}{ Exclusion criteria } \\
\hline & Enlarged internal organs or other signs of storage disease. \\
\hline 2. & Retinopathy, optic atrophy or cataracts. \\
\hline 3. & Evidence of peri- or postnatal brain damage. \\
\hline 4. & Presence of identifiable metabolic disorders or progressive neurological conditions. \\
\hline 5. & Development of neurological disorders as a result of serious infections or head injuries. \\
\hline
\end{tabular}

Girls with RS are born apparently healthy and with normal psychomotor development until the first or second year of life, when brain functional regression begins, with a loss of manual motor skills and the appearance of stereotyped movements, gait apraxia, loss of verbal and nonverbal communication skills, and the association of episodes of hyperventilation, aerophagia and epileptic seizures (8).

There is no specific treatment for RS, apart from physical and occupational therapies to develop and maintain muscle tone and function, together with a range of drugs used to control the seizures (9).

Oral manifestations of RS have been only briefly described in the medical literature, and the dental literature offers few articles on the oral pathology of these patients. The present study offers a systematic review of the literature on the oral manifestations of this rare syndrome.

\section{Search Method}

A Medline/PubMed search (http://www.ncbi.nlm.nih. gov/PubMed) of the literature on the subject published up until 2009 november has been made, based on the following keywords: Rett syndrome AND oral manifes- tation OR dental OR bruxism. The articles were examined on an independent basis by two reviewers (CFG, FJS). The inclusion criteria were: articles in English generated by the Medline database, contributing at least one clinical case with oral manifestations, and describing the buccodental characteristics of the disorder and the dental treatment provided. Of the 363 articles found, only 7 satisfied these criteria.

\section{Review}

The first article on RS published in the dental literature (10) described the case of a 12-year-old girl seen for endodontic treatment of the upper central incisors following damage as the result of a fall in the course of an epileptic attack. The patient had previously undergone dental treatment under general anesthesia. In this case dental treatment was likewise carried out under general anesthesia with nasotracheal intubation. This was followed by dental revision every 6 months.

Pagnacco et al. (11) in turn presented the case of a 5-year-old girl with caries of the deciduous dentition, dental wear and abundant sialorrhea. General anesthesia was used for dental treatment, due to the impossibility of providing care on an outpatient basis. 
Buccino et al. (9) reported the case of a 4-year-old girl presenting stereotyped movements of the hands, hyperventilation, autistic behavior and motor development problems. There were no particular oral features apart from a high-arched palate and the consequences of bruxism, with generalized wear of the deciduous dentition (particularly of the upper and lower anterior sector teeth). In this context, the right and left maxillary incisors showed class II mobility apparently related to the existing bruxism and chronic digital sucking habit. The $\mathrm{X}$-ray study in turn showed external root reabsorption of both incisors, periodontal widening and signs of calcium metamorphosis. A Molt mouth prop was used for dental exploration of the patient, who presented autistic-type behavior with episodes of eye contact with the dental surgeon. The prevention of oral disease was the only recommendation made, establishing the control of bruxism and traumatisms, improving oral hygiene with the help of the parents, the daily application of topical fluor gel or rinses, and frequent follow-up visits, in order to avoid the need for sedation or general anesthesia in posterior dental treatments.

Peak et al. (12) published the case of a 5-year-old girl with RS who presented important wearing of the deciduous dentition, as a result of constant bruxism. At extraoral level she presented bilateral masseter muscle hypertrophy, while at intraoral level the deciduous incisors and canines showed wear. The proposed dental management coincides with the techniques used in application to the disorders seen in autism: the establishment of a routine, minimization of the patient waiting time before entering the dental office, and the avoidance of noise that might frighten the patient during the exploration. Likewise, due to the difficulty of providing dental treatment under local anesthesia, the use of analgesia, sedation and even general anesthesia is recommended where necessary.

The largest RS patient series presented in one same study in the literature comprised 17 cases (13), with a mean age of 7.33 years (range 2.7-12.7 years). At the time of the exploration, 6 of the 17 children presented deciduous dentition, 10 mixed dentition and one permanent dentition. As regards the oral habits, all patients presented digital-manual sucking or nibbling activities, $82 \%$ suffered bruxism, $41 \%$ showed oral breathing, $29 \%$ exhibited tongue thrusting, $29 \%$ suffered dribbling, and $12 \%$ showed lateralization of the mandible. In relation to the oral manifestations, gingivitis with important plaque accumulation was observed in all the patients, probably as a result of parent difficulties in maintaining adequate oral hygiene in the children. Non-physiological dental wear was noted in $71 \%$ of the cases, and was more common in the anterior deciduous teeth, though in contrast only $2.7 \%$ of the explored dental surfaces showed caries. A high-arched palate was present in 53\% of the girls, and a high prevalence of open bite was noted $(80 \%)$ - this possibly being related to the digital-manual sucking and oral breathing. However, the authors of the mentioned series concluded that with the exception of the digital-manual sucking or nibbling activities, there were no pathognomonic oral manifestations of RS. Nevertheless, certain characteristics did prove characteristic, such as bruxism (dental wear), open bite, a high-arched palate, and gingivitis.

The clinical case published by Alpoz et al. (14) in turn corresponded to a 5-year-old girl with day-time bruxism as the sole oral manifestation. This habit resulted in intense dental wear, with almost complete loss of the dental crown, though without pulp exposure. Radiographically, enlargement of the temporomandibular joint space was noted, probably as a result of the bruxism. The patient was subjected to nitrous oxide sedation, and alginate impressions were obtained in order to prepare a soft splint for the upper jaw, to be worn only when awake and controlled by the parents, since the patient did not suffer nocturnal bruxism. This was the only treatment of choice, since it proved impossible to apply other options such as management of the occlusal interferences or the placement of stainless steel crowns (due to scant retention of the deciduous molars).

Magalhaes et al. (15) studied 13 patients with a mean age of 9 years, and with daytime bruxism as the sole oral manifestation in all but two cases. This habit resulted in intense dental wear, and according to the mothers was associated with increased stereotypic movements of the hands. Clenching began by opening of the mouth, followed by mandibular thrusting and closing of the mouth. During bruxism, the tongue was projected towards the anterior teeth, giving rise to anterior open bite in the older patients, in addition to excessive dental wear and muscle dysfunction. For the treatment of bruxism, the authors prepared modified splints placed in the upper jaw so that the cuspids of the deciduous lower molars showed centric occlusion with the splint. The margin of the splint extended to the hard palate, and the use of denture adhesive was recommended to increase retention. A groove measuring $5 \mathrm{~mm}$ in width and $1.5 \mathrm{~mm}$ in depth was made in the occlusal surface of the splint, and was filled with soft and resilient material, in order to avoid anterior mandibular movement. This treatment in turn was combined with acupuncture (reported to be effective in treating temporomandibular dysfunction and facial pain) performed on a weekly basis for an average of three years and four months. The parents reported that bruxism did not reappear until 7 days after the acupuncture sessions, and was moreover of lessened intensity. This combination of modified splints and acupuncture for the treatment of bruxism proved effective in three patients with RS. Likewise, for dental treatment, the authors suggested brief visits, the 
avoidance of physical restraints, and the use of mouth props - since the patients had little or no muscle control.

Among the RS studies published to date, we identified 5 articles involving a single clinical case (9-15), two articles respectively reporting samples of 17 (13) and 13 patients (15), and another article on the orofacial effects and possible interactions of the drugs used to treat the disease (16).

The mean age of the patients described in the reviewed clinical cases and series (9-15) was almost 7 years (specifically 6.8 years), with a range of 2.7-21 years. In the reviewed dental literature, only 35 children with this rare syndrome were subjected to oral exploration - this sample being too small to draw firm conclusions regarding the degree of oral pathology and the problems posed by clinical dental management.

\section{Discussion}

Two groups of oral manifestations of Rett syndrome (RS) can be distinguished: those derived from the medication used to treat the disease on one hand, and the manifestations derived from the specific conditions of RS on the other.

The drug-related (anxiolytic and anticonvulsivant) orofacial manifestations of RS are the following (16): xerostomia, stomatitis, glossitis, erythema multiforme, sialorrhea, dysgeusia, gingivitis, parotid gland swelling, periodontal abscesses, sinusitis, dysphagia and tongue paralysis. However, these manifestations are very difficult to evaluate in patients with RS, unless they prove evident in the course of the clinical exploration. This is particularly due to the communication problem, the lack of language in most cases, and a heightened pain threshold that can mask the presence of pain-producing conditions. On the other hand, consideration is required of the possible interactions between the anxiolytic and anticonvulsivant medication and analgesic drugs widely used in dental practice, such as paracetamol, which at high doses and over the long term lessens the effect of lamotrigine. Likewise, acetylsalicylic acid (aspirin) can inhibit the metabolism of valproic acid, which in turn increases the bleeding tendency associated with the administration of aspirin and other nonsteroidal antiinflammatory drugs. The use of antibiotics must also be taken into consideration. In this sense, carbamazepine accelerates the metabolism of doxycycline, and its own metabolism can be inhibited by erythromycin and clarithromycin. Therefore, a detailed clinical history must be compiled, including all the drugs used by the patient for control of the disease, with a view to avoiding possible undesirable side effects.

The orofacial manifestations and habits of patients with RS found in the literature are described in Table 2. Evaluation of these oral manifestations indicates that the oral findings in RS are not exclusive of the syndrome, since they can also be seen in other diseases characterized by the presence of excessive oral / digital-manual habits, walking problems, seizures, and difficulties for correct oral hygiene $(9,12,13)$. Nevertheless, there are some features, such as bruxism, anterior open bite, a high-arched palate and gingivitis, that are more prevalent in RS. Although bruxism is the oral habit most frequently associated with RS (9,11-16), its treatment remains the subject of controversy. Some authors contraindicate the use of splints in RS due to the important mental retardation of the affected patients, their deficient oral motor control and excessive oral-digital habits (9). In turn, Peak et al. (12) consider that splints should not be used due to the young age of the patients, who still present deciduous dentition. On the other hand, Alpoz et al. (14) have treated bruxism with soft splints, and special designs have even been developed in order to avoid the anterior mandibular movement that accompanies bruxism, combining such splints with acupuncture (15) - though the success obtained is limited.

Table 2. Oral manifestations of Rett syndrome found in the dental literature.

\begin{tabular}{|l|c|c|c|c|}
\hline \multicolumn{1}{|c|}{ Authors } & $\begin{array}{c}\text { Publication } \\
\text { year }\end{array}$ & $\begin{array}{c}\text { Cases } \\
\text { (n) }\end{array}$ & $\begin{array}{c}\text { Age } \\
\text { (years) }\end{array}$ & Oral manifestations \\
\hline Di Bona (10) & 1985 & 1 & 12 & Traumatisms \\
\hline Pagnacco et al. (11) & 1988 & 1 & 5 & Caries, dental wear, sialorrhea \\
\hline Buccino et al. (9) & 1989 & 1 & 4.3 & High-arched palate, bruxism, digit sucking \\
\hline Peak et al. (12) & 1992 & 1 & 5 & Bruxism, masseter hypertrophy \\
\hline Ribeiro et al. (13) & 1997 & 17 & $\begin{array}{c}\mathrm{x}=7.33 \\
(2.7-12.7)\end{array}$ & $\begin{array}{c}\text { Digital-manual sucking / nibbling, bruxism, } \\
\text { oral breathing, tongue thrusting, dribbling, } \\
\text { mandibular lateralization, gingivitis, caries, } \\
\text { high-arched palate, open bite }\end{array}$ \\
\hline Alpoz et al. (14) & 1999 & 1 & 5 & Bruxism \\
\hline Magalhaes et al. (15) & 2002 & 13 & $\begin{array}{c}\mathrm{x}=9 \\
(3-16)\end{array}$ & Bruxism \\
\hline
\end{tabular}


The literature describes the use of mouth props to secure a degree of stability and oral opening, in order to facilitate dental exploration and/or treatment $(9,15)$. As regards behavioral management, some authors (12) have suggested the use of certain techniques used in autism (routines and the avoidance of noise). In this context, consideration is required of the important alteration and limitation in patient communication capacity, the profound mental retardation, and lack of attention. As a result, the dental management techniques must be adapted to each individual case. Some authors have used sedation with nitrous oxide (14), while others combine it with sublingual midazolam (16). In turn, some investigators use general anesthesia $(10,11)$, due to the medical risk of the patient and the lack of cooperation needed for correct dental treatment (17). In any case, all authors agree that prevention, with diet control, the application of fluor and frequent revisions are crucial for the management of these patients, and can limit the use of sedation and general anesthesia to only the most extreme cases.

In conclusion, the present review shows that the deleterious digital-oral habits and orofacial motor functional disorders of these patients must be taken into account, since in the early stages of the disease they can give rise to malocclusions such as anterior open bite and a higharched palate. However, the most characteristic clinical feature of RS is bruxism, of an uncertain etiopathogenesis, and difficult to treat (Fig. 1).

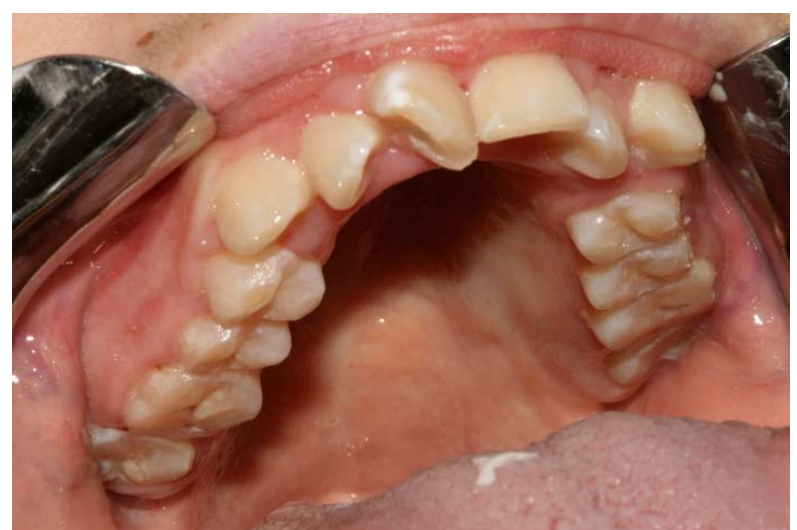

Fig. 1. Clinical characteristics in Rett síndrome: Gingivitis, bruxism and dental fracture.

\section{References}

1. Chahrour M, Zoghbi HY. The story of Rett syndrome: from clinic to neurobiology. Neuron. 2007;56:422-37.

2. Rett A. On a unusual brain atrophy syndrome in hyperammonemia in childhood. Wien Med Wochenschr. 1966;116:723-6.

3. Hagberg B, Aicardi J, Dias K, Ramos O. A progressive syndrome of autism, dementia, ataxia, and loss of purposeful hand use in girls: Rett's syndrome: report of 35 cases. Ann Neurol. 1983;14:471-9.

4. Raizis AM, Saleem M, MacKay R, George PM. Spectrum of MECP2 mutations in New Zealand Rett syndrome patients. N Z Med J. 2009;122:21-8.

5. Guy J, Gan J, Selfridge J, Cobb S, BirdA. Reversal of neurological defects in a mouse model of Rett syndrome. Science. 2007;315:1143-7. 6. Hagberg B, Hanefeld F, Percy A, Skjeldal O. An update on clinically applicable diagnostic criteria in Rett syndrome. Comments to Rett Syndrome Clinical Criteria Consensus Panel Satellite to European Paediatric Neurology Society Meeting, Baden Baden, Germany, 11 September 2001. Eur J Paediatr Neurol. 2002;6:293-7.

7. Pineda M, Aracil A, Vernet A, Espada M, Cobo E, Arteaga R, et al. Rett's syndrome in the Spanish population. Rev Neurol. 1999;28:105-9.

8. Budden SS, Dorsey HC, Steiner RD. Clinical profile of a male with Rett syndrome. Brain Dev. 2005;27 Suppl 1:S69-S71.

9. Buccino MA, Weddell JA. Rett syndrome--a rare and often misdiagnosed syndrome:case report. Pediatr Dent. 1989;11:151-7.

10. Di Bona MC. Hospital dentistry for a child with Rett's syndrome. Spec Care Dentist. 1985;5:62-3.

11. Pagnacco A, Ferrara M, Vangelisti R, Randon C. Rett syndrome: a "new" and rare disease. A clinical case. Dent Cadmos. 1988;56:93-5.

12. Peak J, Eveson JW, Scully C. Oral manifestation of Rett's syndrome. Br Dent J.1992;172:248-9.

13. Ribeiro RA, Romano AR, Birman EG, Mayer MP. Oral manifestations in Rett syndrome: a study of 17 cases. Pediatr Dent. 1997;19:34952.

14. Alpoz AR, Ergul N, Oncag O. Bruxism in Rett syndrome: a case report. J Clin Pediatr Dent. 1999;23:161-3.

15. Magalhães MH, Kawamura JY, Araújo LC. General and oral characteristics in Rett syndrome. Spec Care Dentist. 2002;22:147-50.

16. Friedlander AH, Yagiela JA, Paterno VI, Mahler ME. The pathophysiology, medical management and dental implications of fragile $X$, Rett, and Prader-Willi syndromes. J Calif Dent Assoc. 2003;31:693702 .

17. Escribano Hernández A, Hernández Corral T, Ruiz-Martín E, Porteros Sánchez JA. Results of a dental care protocol for mentally handicapped patients set in a primary health care area in Spain. Med Oral Patol Oral Cir Bucal. 2007 Nov 1;12(7):E492-5. 